\title{
La relation emploi - innovation dans les services
}

The relationship between innovation and employment in service

\section{Faridah Djellal et Faïz Gallouj}

\section{CpenEdition}

\section{Journals}

Édition électronique

URL : http://journals.openedition.org/travailemploi/4860

DOI : 10.4000/travailemploi.4860

ISSN : 1775-416X

\section{Éditeur}

DARES - Ministère du Travail

\section{Édition imprimée}

Date de publication : 15 octobre 2006

Pagination : 45-56

ISSN : 0224-4365

\section{Référence électronique}

Faridah Djellal et Faïz Gallouj, « La relation emploi - innovation dans les services », Travail et Emploi [En ligne], 108 | octobre-décembre 2006, mis en ligne le 15 décembre 2008, consulté le 04 mai 2019. URL http://journals.openedition.org/travailemploi/4860 ; DOI : 10.4000/travailemploi.4860 


\title{
La relation innovation emploi dans les services
}

\author{
Faridah Djellal, Faïz Gallouj ( ${ }^{*}$
}

La question de l'innovation dans les services et de ses relations à l'emploi est une question fondamentale, qui paradoxalement n'occupe pas la place qui lui revient dans la littérature économique. Cet article tente, à titre essentiellement programmatique, d'articuler la question de l'emploi aux principaux travaux empiriques ou théoriques qui ont été consacrés à l'innovation dans les services. Bien qu'unanimement considérée comme fondamentale, cette question est relativement peu exploitée: en effet, l'une des variables de la relation (à savoir la question de l'innovation dans les services) n'est pas encore clairement élucidée sur le plan théorique. Le caractère encore flou de la nature et du statut de l'innovation dans les services conduit à considérer comme hasardeuse toute tentative d'analyse des conséquences de cette innovation sur l'emploi. Il s'agit-là pourtant d'un champ de recherche important sur le plan théorique et des politiques publiques qu'il faudrait explorer davantage, à la fois sur le plan micro, méso et macroéconomique, que ce soit par le biais de la construction théorique déductive ou de l'analyse inductive qualitative ou statistique. Ce sont les deux axes généraux de recherche que l'article explore, dans une volonté de fournir un premier cadrage pour une réflexion à développer.

La question de la relation entre le changement technique et l'emploi est une question ancienne et fondamentalement complexe, à la fois sur le plan théorique et empirique, indépendamment même du problème du secteur concerné (FreEman, Soete, 1987; Petit, 1995; Vivarelli, 1995). Elle renvoie à des causalités multiples et contradictoires, directes et indirectes. Elle ne semble pas pouvoir être abordée de manière satisfaisante par l'intermédiaire d'un nombre réduit de mécanismes généraux, ni à un seul niveau d'analyse, micro, méso ou macroéconomique. Les débats autour de la «théorie de la compensation» (selon laquelle les ressorts du marché sont en mesure de compenser, de manière automatique, les pertes d'emplois induites par une innovation destructrice d'emplois(1)) donnent une idée de la complexité des mécanismes à l'œuvre. L'écheveau des relations est par ailleurs rendu encore plus inextricable par le fait que d'autres variables que l'innovation interviennent sur la croissance de l'emploi dans les services: la demande, les changements institutionnels, etc. Il n'est donc pas étonnant, comme le soulignent Vivarelli et Pianta (2000) que l'économie néoschumpeterienne contemporaine de l'innovation (à l'exception notable des travaux de Freeman, Clark, Soete, 1982; Freeman,

\footnotetext{
*Clersé, Ifrési et université de Lille 1; faiz.gallouj@univ-lille1. fr; faridah.djellal@univ-lille1.fr

(1) On considère généralement que la compensation opère à travers les différents mécanismes suivants: la création de nouvelles machines, la baisse des prix, de nouveaux investissements, la baisse des salaires, l'augmentation des revenus, la création de nouveaux produits (Vivarelli, 1995 ; Petit, 1995).
}

SoEte, 1987) ait évité cette question. Il l'est encore moins qu'elle soit généralement absente des travaux consacrés à l'innovation dans un secteur qui pose de nombreux autres problèmes théoriques intéressants et difficiles (en particulier ceux de la définition, de la mesure, des modes d'organisation et d'appropriation de l'innovation et de la R-D).

Ainsi, bien que le secteur des services soit reconnu comme le principal créateur d'emplois dans les économies contemporaines, trop peu de travaux se sont penchés sur la question du lien entre l'innovation dans ce secteur et l'emploi. Ce désintérêt pour une question théorique importante et ancienne peut être interprété de différentes manières. Tout d'abord, dans la mesure où la littérature économique a longtemps sous-estimé l'innovation dans les services, il n'est pas étonnant qu'elle ait également sous-estimé et négligé ses effets sur l'emploi. Ensuite, la loi d'Engel et la loi de la productivité ont fourni des arguments théoriques satisfaisants pour expliquer la croissance des services et de l'emploi dans les services (Fourastié, 1949; BAUMOL, 1967; Bell, 1976). En effet, selon la première de ces lois, la demande et la consommation de services augmentent dans des économies dont les revenus sont croissants, et selon la seconde, le faible taux de croissance de la productivité dans les services, y entraîne un accroissement de l'emploi. Les principales théories de la croissance tertiaire ont ainsi écarté l'argument de l'innovation dans les services (ou se sont contentées du constat de la faiblesse de l'innovation dans ce secteur), pour expliquer le glissement inéluctable d'une économie industrielle à une économie de services. Enfin, les travaux, qui, 
en particulier aux États-Unis, se sont efforcés de dénoncer la mauvaise qualité des emplois engendrés par l'économie des services ont également contribué, à leur manière, à occulter la question du lien innovation-emploi. En effet, sous l'angle de la création d'emplois, la société de service ne serait rien d'autre qu'une "société de serviteurs», une hamburger society, une bad jobs society, essentiellement génératrice de «McJobs» (Bluestone, HARRISON, 1986; Thurow, 1989; MaHAR, 1992). Il est peu probable qu'une telle société soit propice à l'innovation.

Notre objectif, dans cet article, est de proposer une relecture, à la lumière de la question de l'emploi, des travaux consacrés à l'innovation dans les services. Ces travaux sont souvent divisés en deux groupes distincts (GallouJ, 1994). Le premier groupe (les approches technologistes) est constitué de travaux qui réduisent, pour l'essentiel, l'innovation dans les services à l'adoption de systèmes techniques, tandis que le second groupe (les approches servicielles), réunit les travaux qui mettent l'accent, en particulier, sur les formes non technologiques de l'innovation.

Cet article se propose d'évaluer dans quelle mesure et sous quelle forme la question de l'emploi est implicitement ou explicitement présente ou mérite d'être introduite dans les deux groupes ainsi identifiés. Il s'agit donc de rendre compte, que ce soit au niveau de la firme, du secteur ou au niveau macro-économique, de la façon dont la question de l'emploi s'articule (ou peut s'articuler) avec celle de l'innovation, lorsqu'il s'agit des services. Ce projet constitue à la fois un bilan de la littérature et un agenda de recherche. À ce titre, il pose beaucoup plus de questions qu'il n'apporte de réponses.

\section{La question de l'emploi au cœur des approches technologistes}

Les travaux identifiant l'innovation dans les services à l'innovation technologique (adoptée par les services) sont, de loin, les plus anciens et les plus nombreux, ce qui contribue, dans une certaine mesure, à sous-estimer d'autres aspects de l'innovation. Au-delà des interprétations théoriques (notamment les hypothèses associées au concept de fonction de production, qui accorde une place centrale aux innovations de processus), le principal argument qui peut justifier une telle orientation des travaux de recherche est que les services sont des secteurs de plus en plus intensifs en technologies et en capital.

On peut décomposer cet axe technologiste en trois groupes de travaux, différents par leur degré d'ambition théorique, le type d'innovation technologique qu'ils privilégient, et disproportionnés quant à leur taille. Le premier groupe est consacré aux consé- quences de l'introduction de systèmes techniques dans les firmes ou secteurs de services (analyses en termes d'impacts). Nous distinguerons ici les analyses en termes d'impacts des TIC des analyses plus générales en termes d'impacts de l'innovation technologique quelle qu'elle soit. Le second groupe ne comporte qu'une seule référence (le modèle du cycle de vie inversé de Barras), qui a pour ambition de proposer une théorie de l'innovation dans les services. Le dernier groupe est constitué de travaux d'inspiration évolutionniste, qui visent à retracer des trajectoires d'innovation dans les services. Seuls les deux premiers groupes envisagent explicitement la question de l'emploi. Bien que les approches taxonomiques initiales (celles de Pavitt ou de Soete et Miozzo) n'abordent pas explicitement cette question, on peut considérer qu'elle y est implicitement présente ou qu'elle peut y être introduite, dans une certaine mesure, à travers les implications en termes d'emplois des formes d'innovation et de la nature des trajectoires envisagées.

\section{L'impact des technologies informationnelles sur l'emploi dans les services}

Une part considérable de la littérature consacrée à l'innovation dans les services s'intéresse aux conséquences de la diffusion des technologies informationnelles aux niveaux micro, méso et macro-économiques (Bertrand, Noyelle, 1987; Cossalter, HÉZARD, 1983; DJellal, 2002). Pour simplifier, on peut décrire l'expansion de ces technologies à travers deux modèles d'innovation: l'informatique lourde, d'une part, et l'informatique décentralisée et les réseaux, d'autre part. Une manière commode de rendre compte sinon des résultats du moins des principaux questionnements généraux envisagés dans cette abondante littérature (en termes d'impacts) est de croiser ces deux modèles avec un ensemble de variables économiques fondamentales comme l'emploi, les qualifications et l'organisation des tâches, la productivité, le caractère échangeable $\mathrm{du}$ «service » et sa qualité ( $c f$. figure 1).

Figure 1

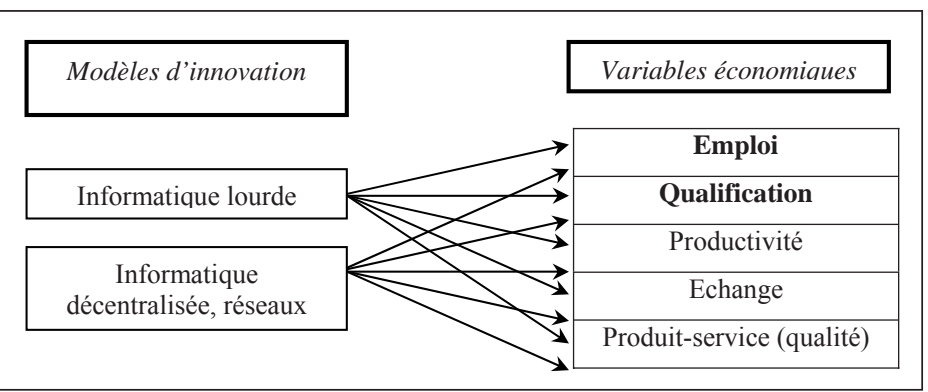

Les analyses en termes d'impacts des TIC

Le modèle de l'informatique lourde aurait ainsi plutôt tendance à exercer un effet positif sur la productivité et l'échange, mais négatif sur l'emploi 
et la qualification de la main-d'œuvre. Ce modèle d'innovation correspond à l'informatisation des back-office (c'est-à-dire des activités qui ne se trouvent pas au contact des clients) et vise avant tout à la réduction du coût de la prestation, par la standardisation des tâches et l'exploitation d'économies d'échelles. Quant au modèle de l'informatique décentralisée et des réseaux, il aurait plutôt tendance à exercer des effets positifs sur l'emploi (ou sur certains types d'emplois), la qualification, la facilité d'échange, mais aussi éventuellement sur la productivité et la qualité du "produit». Ce second modèle modifie le front-office (c'est-à-dire l'interface avec les clients). Il engendre des économies de variété et réduit les tâches routinières en faveur d'activités commerciales et de conseil.

Les réflexions précédentes ne constituent pas des réponses ou des résultats définitifs, mais plutôt des hypothèses. Quel que soit le modèle d'innovation considéré, une partie importante de la littérature consiste à présenter l'une, plusieurs ou l'ensemble de ces hypothèses théoriques et leurs mécanismes, à les confronter à la réalité et à tenter d'interpréter les écarts éventuels.

Quel que soit le modèle considéré, la question de l'innovation dans les services peut être abordée sous l'angle de ses conséquences sur d'autres dimensions qualitatives de l'emploi. On peut se poser la question de ses conséquences sur la distribution sexuelle de l'emploi. Un certain nombre de travaux (APPELBAUM, 1987; WeBSTER, 1996) suggèrent qu'en ce qui concerne l'emploi, l'innovation n'est pas sexuellement neutre, autrement dit qu'elle est biaisée selon le genre. ApPelBaum (1987) étudie la manière dont l'innovation modifie la structure de l'emploi dans le secteur de l'assurance de sorte que des fonctions qui étaient auparavant masculines se féminisent ( $c f$. aussi WeBster, 1986).

D'autres questions intéressantes sont, à notre connaissance, peu présentes dans la littérature. Il s'agit en particulier des conséquences de l'innovation dans les services sur ce que DevetTer (2001) appelle la disponibilité temporelle au travail, qu'on pourrait élargir à la disponibilité spatio-temporelle au travail. Certaines formes d'innovation technologique ou organisationnelle dans les services facilitent ou induisent des formes de travail à temps partiel, de dilution du temps de travail dans le temps domestique, ou des formes de mobilisation $\mathrm{du}$ travailleur dans des espaces spatio-temporels atypiques: le domicile dans le cas du télétravail, de l'usage de la téléphonie mobile, de l'ordinateur portable et de l'Internet.

C'est probablement dans ce groupe de travaux que la question de la relation innovation qualification est le plus souvent abordée. Il existe néanmoins une littérature beaucoup plus abondante centrée sur la thématique de la qualification des emplois tertiaires. Nous n'en rendons pas compte ici dans la mesure où la question de l'innovation en est absente ou n'y occupe qu'une place incidente. Nous avons d'ores et déjà cité en introduction un certain nombre de travaux essentiellement américains sur ce thème. Mais il existe également de nombreux travaux français (BERTRAND, 1988; JANY-CATRICE, 2004; GADREY, 2005). Bertrand propose ainsi une typologie des emplois de service qui distingue, sur la base de critères qualitatifs relatifs à l'emploi, les trois groupes suivants : 1) le tertiaire technico-administratif et socio-culturel; 2) les services commerciaux et personnels; 3) le tertiaire "para-industriel».

Pour saisir dans toute leur complexité les relations entre NTIC, services et emplois, on ne peut pas se contenter d'une analyse des NTIC comme facteur exogène (analyses en termes d'impacts). On assiste en effet à une endogénéisation croissante des NTIC dans l'économie des services. Les services ne sont plus ainsi simplement envisagés à travers leurs comportements d'adoption de ces NTIC. Ils peuvent jouer un rôle de plus en plus actif dans leur production et leur diffusion; et l'innovation de service apparaît souvent comme une catégorie hybride associant des NTIC et une activité d'ingénierie organisationnelle, c'est-à-dire de conception-développement de formules organisationnelles (Duellal et alii, 2003). Cette endogénéisation des NTIC est à l'origine de nouveaux produits-services, de nouvelles demandes, de nouvelles activités et de nouveaux marchés, et par conséquent de nouveaux emplois (Petit, Soete, 2001).

\section{L'impact de l'innovation technologique (sous toutes ses formes) sur l'emploi dans les services}

Tirant parti des enquêtes communautaires sur l'innovation (ECI), un certain nombre de travaux statistiques récents et en nombre restreint (EvANGELISTA, 2000a; Evangelista, Savona, 2003; Nählinder et Hommen, 2002; Peters, 2004) se sont efforcés de mesurer les effets de l'innovation sur l'emploi et les qualifications dans les services ( $c f$. tableau 1).

La version italienne de 1'ECI 2, réalisée en 1997 pour la période 1993-1995, a ainsi collecté des données sur les impacts de l'innovation sur l'emploi total des entreprises de manière globale (la question posée étant: l'introduction de l'innovation a-t-elle conduit 1) à un accroissement de l'emploi; 2) à une baisse de l'emploi; 3) à aucun impact sur l'emploi?), puis, en dissociant trois niveaux de qualification «élevé, moyen, faible». Les questions et les données correspondantes ne sont pas associées à chacun des types d'innovation pris en compte par le questionnaire (à savoir: la création d'un nouveau service, l'amélioration d'un service existant, l'introduction d'un nouveau process). Ainsi, les réponses obtenues sont des «résultats» nets (de la somme des différents effets). En exploitant cette enquête, 
Tableau 1

\section{L'impact de l'innovation technologique sur l'emploi dans les services}

\begin{tabular}{|c|c|c|}
\hline Enquête & Description du dispositif & Principales conclusions \\
\hline $\begin{array}{l}\text { Italienne } \\
\text { EVANGELISTA } \\
(2000 \mathrm{a})\end{array}$ & $\begin{array}{l}\text { ECI } 2 \text { en } 1997 \text { période 1993-1995 } 6000 \\
\text { entreprises }\end{array}$ & $\begin{array}{l}\text { - Innovation : effet positif sur emploi } \\
\text { - Effet positif très fort pour les emplois très qualifiés } \\
\text { - Effet négatif pour les emplois les moins qualifiés } \\
\text { - Impact variable selon la taille des firmes : } \\
+ \text { grandes entreprises : effet destructeur d'emplois (surtout peu qualifiés) } \\
+ \text { petites entreprises : effet créateur }\end{array}$ \\
\hline $\begin{array}{l}\text { Suédoise } \\
\text { NÄHLINDER } \\
\text { et HOMMEN } \\
(2002)\end{array}$ & $\begin{array}{l}\text { ECI } 2 \text { période } 1994-1996 \\
743 \text { entreprises (dont } 140 \text { firmes de SIC) } \\
\text { L'analyse ne porte que sur les SIC }\end{array}$ & $\begin{array}{l}\text { - Innovation : effet positif sur l'emploi } \\
\text { - Effet positif sur les qualifications }\end{array}$ \\
\hline $\begin{array}{l}\text { Allemande } \\
\text { PETERS } \\
(2004)\end{array}$ & $\begin{array}{l}\text { ECI } 3 \\
\text { réalisée en } 2001 \\
\text { période } 1998-2000 \\
2200 \text { firmes industrielles et de services }\end{array}$ & $\begin{array}{l}\text { - Innovation de produit : impact positif sur l'emploi dans les services } \\
\text { - Innovation de process : pas eu d'effet négatif sur l'emploi dans les } \\
\text { services }\end{array}$ \\
\hline
\end{tabular}

Evangelista (2000a) constate ainsi que l'innovation exerce un effet globalement positif sur l'emploi dans les services. Cet effet positif intervient de manière particulièrement forte sur les emplois très qualifiés, tandis que les effets «destructeurs d'emplois» du changement technique jouent essentiellement sur les composantes les moins qualifiées de l'emploi. L'enquête montre également que l'impact de l'innovation varie selon la taille des firmes. Dans les grandes firmes, l'innovation exerce un effet «destructeur d'emplois» (qui affecte essentiellement les emplois peu qualifiés), tandis que, dans les petites firmes, son effet sur l'emploi est positif.

En s'intéressant aux services intensifs en connaissances (SIC), NÄHLINDER et HOMmEN (2002) confirment cette tendance, pour la Suède, sur la base de 1'ECI 2. Les innovations dans les SIC seraient ainsi positivement corrélées à une croissance de l'emploi comme des qualifications. Ces résultats ne suffisent pas à remettre en cause l'hypothèse récurrente selon laquelle les services seraient essentiellement créateurs d'emplois de faible qualification. Les SIC ne sont pas représentatifs de l'ensemble des services. Compte tenu de leur objet particulier, ils ont tendance (indépendamment même de la relation à l'innovation) à recruter des personnels fortement qualifiés.

Pour l'Allemagne, Peters (2004) s'appuie sur l'ECI 3 pour tenter d'évaluer l'impact sur l'emploi des innovations de produit et de process. La démarche se distingue des précédentes dans la mesure où ces deux types d'innovation ne sont pas envisagés comme des catégories homogènes. L'analyse distingue en effet deux types d'innovation de produit différents selon leur degré de nouveauté (nouveauté pour le marché, nouveauté pour l'entreprise) et deux types d'innovations de processus (d'une part, les technologies de rationalisation, et d'autre part, les innovations de processus qui visent d'autres objets que la rationalisation: par exemple, l'amélioration de la qualité, les exigences réglementaires...). L'analyse économétrique indique que les innovations de produit (qu'il s'agisse de nouveauté pour le marché ou d'imitations, sans différence significative entre les deux) ont un impact net positif sur l'emploi au niveau de la firme. Ce résultat, établi à la fois pour l'industrie et les services, contredit les hypothèses traditionnelles selon lesquelles l'innovation de marché génère davantage d'emplois que l'innovation d'imitation. L'innovation de processus (en particulier sous sa forme d'innovation de rationalisation) exerce des effets négatifs sur l'emploi dans l'industrie. En revanche, durant la période considérée, cette innovation ne semble pas être à l'origine d'une réduction significative de la maind'œuvre, dans les services.

Ces exercices statistiques ne doivent pas nous faire oublier les nombreux problèmes méthodologiques qui en atténuent la portée. En effet, les données des ECI sont obtenues sur la base des indicateurs du Manuel d'Oslo (OCDE, 1997). Or, ces indicateurs sont restrictifs (DJellal, Gallouj, 2000). Ils ne s'intéressent qu'à l'innovation technologique. Un autre problème est celui de la définition de la compétence (et donc de l'appréciation de son évolution positive ou négative au sein d'une entreprise). Les enquêtes ont probablement tendance à sous-évaluer les conséquences positives de l'innovation sur la qualification. Les directives du Manuel de Canberra (OCDE, 1995) qui ne considèrent comme fortement qualifiés que les employés qui ont une formation post secondaire ne paraissent pas satisfaisantes à cet égard.

La question de la distinction entre innovation de produit et innovation de process est ici importante, 
dans la mesure où ces deux formes d'innovation ont, en théorie, des impacts différents sur l'emploi. En effet, l'innovation de processus, dans son acception traditionnelle, consiste à substituer du capital au travail. Elle a donc un effet destructeur d'emplois. L'innovation de produit consiste à introduire des qualités nouvelles sur le marché et donc à susciter une demande nouvelle. Elle contribue à la conquête de nouveaux marchés, autrement dit, à l'accroissement de la production et de l'embauche. Elle est par conséquent créatrice d'emplois. C'est, selon Katsoulacos (1984), cette succession de périodes dominées par l'innovation de produit ou l'innovation de process qui explique les fluctuations du chômage. Même dans l'industrie, cette distinction doit néanmoins être utilisée avec prudence. Ainsi, la nature «produit» ou «process » d'une innovation n'est pas une caractéristique technique intrinsèque. Elle peut dépendre des usages. Dans le domaine des biens d'équipement (celui des machines-outils par exemple), une innovation de produit pour un secteur (le secteur producteur) devient généralement une innovation de process pour un autre secteur (celui qui l'adopte ou l'utilise), avec des effets différents sur l'emploi pour un même système.

Dans les services, cette distinction est difficile à opérer. En excluant les formes non technologiques de l'innovation (dont une bonne partie sont vraisemblablement des innovations non technologiques de produit, par exemple, les produits financiers, les produits d'assurance, il est probable que les régressions économétriques effectuées mettent davantage l'accent sur les innovations de process (des systèmes techniques introduits dans la prestation de service). Par ailleurs, une conception moins matérielle et technologique de l'innovation de process (par exemple, l'innovation méthodologique dans le conseil) peut, dans certains cas, obscurcir la relation innovation-emploi, dans la mesure où l'innovation méthodologique peut avoir des objectifs différents : celui d'économiser du temps en rationalisant et coordonnant les actions et en réduisant les aléas («effet process»), mais aussi celui de conférer un squelette au «produit-service», voire d'ajouter des fonctionnalités, des qualités à un produit (par exemple, un processus de livraison à domicile). C'est, dans ce cas, plutôt l'«effet produit» qui intervient.

\section{Le modèle du cycle inversé et la question de l'emploi}

BARRAS (1986) est à l'origine d'un modèle simple et suggestif qui constitue une théorie de la diffusion de l'innovation technologique d'origine industrielle dans les services. Il décrit le cycle de l'innovation dans les services comme l'inverse du cycle industriel traditionnel formalisé par ABERNATHY et UTTERBACK (1978). Ce cycle inversé, induit par les différentes vagues d'informatisation, articule successivement une phase d'innovation de process incrémentale, une phase d'innovation de process radicale et une phase d'innovation de produit ( $c f$. tableau 2).

$$
\text { Tableau } 2
$$

\section{Le cycle du produit inversé, un cycle de l'emploi}

\begin{tabular}{|l|l|l|}
\hline $\begin{array}{l}\text { Étapes } \\
\text { du cycle }\end{array}$ & $\begin{array}{l}\text { Forme d'inno- } \\
\text { vation domi- } \\
\text { nante }\end{array}$ & $\begin{array}{l}\text { Impacts du progrès technique } \\
\text { sur les facteurs de production }\end{array}$ \\
\hline $\begin{array}{l}\text { Première } \\
\text { étape }\end{array}$ & $\begin{array}{l}\text { Innovation } \\
\text { de process } \\
\text { incrémentale }\end{array}$ & $\begin{array}{l}\text { Progrès technique qui épargne } \\
\text { du travail et augmente la } \\
\text { quantité de capital utilisée }\end{array}$ \\
\hline $\begin{array}{l}\text { Deuxième } \\
\text { étape }\end{array}$ & $\begin{array}{l}\text { Innovation } \\
\text { de process } \\
\text { radicale }\end{array}$ & $\begin{array}{l}\text { Progrès technique neutre vis- } \\
\text { à-vis du travail et favorisant } \\
\text { l'augmentation de la quantité } \\
\text { et de la qualité et du capital }\end{array}$ \\
\hline $\begin{array}{l}\text { Troisième } \\
\text { étape }\end{array}$ & $\begin{array}{l}\text { Innovation } \\
\text { de produit }\end{array}$ & $\begin{array}{l}\text { Progrès technique augmentant } \\
\text { la qualité du capital et épar- } \\
\text { gnant celui-ci (utilisant du } \\
\text { travail) }\end{array}$ \\
\hline
\end{tabular}

Les innovations de process incrémentales apparaissent dans le back-office des organisations. Elles obéissent à une logique d'efficience et de réduction des coûts. Il s'agit, par exemple, de l'enregistrement informatisé des polices d'assurance, de l'informatisation des registres du personnel et des salaires. Dans cette première phase, le cycle inversé est caractérisé par un progrès technique qui épargne le travail et augmente la quantité de capital. Dans les secteurs de services caractérisés par une forte croissance de la demande, le biais «destructeur d'emplois » du progrès technique peut être masqué. Cependant, comme le note BARRAS (1986), c'est dans les secteurs affectés par une saturation, voire un rétrécissement des marchés, que la tendance à l'introduction de technologies destructrices d'emplois est la plus forte.

Les innovations de process radicales concernent essentiellement le front office et elles visent l'amélioration de la qualité. Il s'agit, par exemple, des enregistrements «en direct» des polices dans les bureaux des compagnies d'assurance, de l'installation de guichets automatiques de banque. L'amélioration de la qualité induit une certaine extension des marchés. Dans cette seconde phase du cycle, le progrès technique (en valeur nette) est plus neutre quant au facteur travail et il a un effet équivalent d'augmentation de la qualité et de la quantité du capital.

Les innovations de «produit» quant à elles sont encore relativement rares. La banque à domicile en est la meilleure illustration. Ces innovations visent moins l'efficacité ou la différenciation des produits que la conquête de nouveaux marchés. Elles ont donc un effet positif sur l'emploi. Elles sont associées à un progrès technique qui augmente la qualité du capital tout en épargnant celui-ci, c'est-à-dire en utilisant du travail. 
On constate ainsi que le cycle inversé du produit est aussi un cycle de l'emploi : dans les services, l'innovation commence par être destructrice d'emplois avant d'en être créatrice mais, le modèle du «cycle inversé» et ses implications sur l'emploi achoppent sur sa conception particulière du produit et de la distinction entre produit et process. Par ailleurs, qu'elle soit de produit ou de process, l'innovation envisagée par Barras est une innovation technologique. Ainsi, certaines formes de l'innovation sont exclues du modèle, alors qu'on peut faire l'hypothèse qu'elles exercent un important effet «créateur d'emplois». C'est le cas de l'innovation de produit envisagée, par exemple, dans la banque et l'assurance, dans le sens d'un nouveau produit financier ou d'un nouveau contrat. Le biais technologique du cycle inversé de Barras introduit donc un biais dans l'analyse des impacts de l'innovation sur l'emploi.

\section{Les approches taxonomiques}

Les approches en termes de taxonomies sectorielles ont, a priori, l'intérêt de pouvoir permettre de déplacer l'analyse au niveau sectoriel, et par conséquent de mieux appréhender certains phénomènes de «compensation». Mais, ces taxonomies ne s'intéressent pas explicitement aux impacts des différentes trajectoires d'innovation sur l'emploi. Ainsi, par exemple, dans la taxonomie de SoEte et Miozzo (1990), comme dans celle de PavitT (1984), la taille de la firme (évaluée par ses effectifs) est un des déterminants structurels et non une variable dépendante de l'évolution le long de la trajectoire.

Cependant, dans la mesure où ces taxonomies s'appuient sur la distinction fondamentale entre innovation de produit et innovation de process, et sur la part relative de chacun de ces types dans un secteur donné, on peut faire l'hypothèse que, toutes choses égales par ailleurs, les trajectoires sectorielles correspondantes où domine l'innovation de produit sont créatrices d'emplois, tandis que les trajectoires sectorielles dominées par l'innovation de process sont destructrices d'emplois. On constate ainsi que, dans la taxonomie de Pavitt, tous les services appartiennent à la catégorie "dominée par les fournisseurs ", ce qui signifie qu'ils adoptent des innovations de process élaborées dans d'autres secteurs. Toutes choses égales par ailleurs, l'innovation dans les services est ainsi envisagée comme ayant un effet «destructeurs d'emplois», ce qui est problématique (même si des effets de compensations peuvent être en jeu et que d'autres facteurs que l'innovation interviennent) au regard des statistiques de l'emploi. La taxonomie de Soete et Miozzo (qui distingue, rappelons-le, dans le domaine des services: 1) les firmes dominées par les fournisseurs; 2) les réseaux physiques; 3) les réseaux informationnels; 4) les fournisseurs spécialisés et fondés sur la science) est, de ce point de vue, plus satisfaisante dans la mesure où elle introduit aussi des trajectoires où l'innovation de produit est domi- nante. C'est le cas, par exemple, des fournisseurs spécialisés et fondés sur la science.

Pour intéressantes qu'elles soient ces taxonomies sectorielles sont abstraites. Evangelista (2000b) ainsi que Evangelista et Savona (2003), en s'appuyant sur un certain nombre d'indicateurs issus de l'ECI 2 (pour l'Italie voir tableau 1), proposent une taxonomie sectorielle plus concrète et opérationnelle, qui est en mesure de permettre d'envisager une analyse statistique de la relation innovationemploi au niveau sectoriel.

Cette taxonomie distingue trois modèles sectoriels d'innovation dans les services:

1) les «utilisateurs de technologies». Cette catégorie regroupe les branches les plus traditionnelles des services à savoir le commerce de détail, l'hôtellerie, la restauration, le transport, le nettoyage, etc. Il s'agit de secteurs faiblement innovateurs et dépendants des fournisseurs industriels de technologies;

2) les « utilisateurs de TIC». Ces secteurs sont caractérisés par leur usage intensif des TIC. Il s'agit, en particulier, des banques, des compagnies d'assurance et autres services financiers, du commerce de gros, de la publicité, etc.;

$3)$ les «secteurs fondés sur les sciences et techniques ». On compte dans ce secteur la R-D, l'ingénierie, les conseils techniques, les services informatiques. Ce sont des secteurs caractérisés par leur forte activité d'innovation.

Le travail statistique concernant l'impact de l'innovation sur l'emploi effectué sur la base de cette taxonomie aboutit aux résultats suivants:

1) dans les firmes fondées sur les S-T, l'innovation a un effet globalement positif sur l'emploi. On constate par ailleurs une importante tendance à la substitution de la main-d'œuvre très qualifiée à la main-d'œuvre peu qualifiée. Le changement technique a donc un biais de compétence;

2) dans le secteur des utilisateurs de TIC, on constate un effet négatif de l'innovation sur l'emploi ainsi qu'un biais de compétence important. Autrement dit, l'introduction de TIC dans les banques, l'assurance, les services postaux, etc. exerce un effet destructeur d'emplois. Le recrutement d'une main-d'œuvre qualifiée (consécutive à l'introduction de nouveaux services) n'a pas été en mesure de compenser la destruction d'emplois de faible qualification;

3) dans le secteur des utilisateurs de technologies, l'innovation (c'est-à-dire l'adoption de nouveaux systèmes de transports, par exemple, mais aussi de nouveaux systèmes informatiques) a des conséquences négatives (modérées) sur le volume d'emplois ainsi qu'un biais de compétence (modéré). On constate ainsi que la tendance au remplacement d'une main-d'œuvre moins qualifiée par une main-d'œuvre plus qualifiée est une caractéristique générale pour l'ensemble du secteur des services. 
Les questions des modèles nationaux d'économies et de sociétés de services (GADREY, 2005) et de systèmes nationaux de production et d'innovation (AMABLE et alii, 1997) entretiennent d'évidentes relations avec ces typologies des trajectoires technologiques sectorielles. En effet, la nature des trajectoires technologiques et leurs conséquences sur la quantité et la qualité de l'emploi dépendent fondamentalement de la configuration du modèle ou du système, c'est-à-dire en particulier des secteurs qu'il privilégie. Ainsi, en mettant l'accent sur les structures quantitatives et qualitatives de l'emploi dans les services, GADREY (2005) met en évidence quatre «mondes»d'économies et de sociétés de services: libéral, nordique, continental et familialiste. Les critères à l'origine de cette typologie sont les suivants : 1) le poids relatif des services personnels et commerciaux, d'une part, et des services sociaux, au sens large, d'autre part;2) la qualité de ces emplois; 3) le poids relatif du marchand et du non-marchand.

Plus généralement, il semble que la relation entre l'innovation et l'emploi (en particulier dans les services) peut difficilement être envisagée indépendamment d'une réflexion sur les régimes de croissance (BOYER, 2002). Dans une réflexion consacrée au potentiel de croissance et de productivité des économies développées contemporaines (économies caractérisées à la fois par un niveau élevé de tertiarisation et des régimes de croissance appuyés sur les vagues des NTIC), Pетіт (2002) considère que deux facteurs importants relatifs au tertiaire jouent un rôle dans l'exploitation de cette nouvelle croissance: la capacité des pays à transformer les services sociaux et les services aux ménages (en particulier par l'innovation, la qualification et la professionnalisation), leur capacité à faire du secteur tertiaire complexe un pôle d'innovation et de support du changement et de l'innovation.

\section{La question de l'emploi absente des approches servicielles}

On distingue en général deux groupes d'approches servicielles : 1) les travaux typologiques consacrés à différents types de services; 2) les théories locales de l'innovation adaptées à des secteurs de service particuliers. La question de l'emploi est rarement abordée par ces travaux, dont les efforts portent essentiellement sur la recherche de spécificités éventuelles de l'innovation dans les services, tant dans sa forme que dans ses modes d'organisation ou sa dynamique historique (GAllou, 2002). Il s'agit ici moins de rendre compte de résultats établis par la littérature, que d'ouvrir un certain nombre de pistes de réflexion méritant d'être explorées.

\section{Roue de la distribution et roue de l'emploi, théorie de l'accordéon et emplois en accordéon?}

Les spécialistes du commerce ont tenté d'élaborer des modèles théoriques permettant de comprendre la dynamique de l'innovation dans ce secteur. Deux modèles ont connu un succès important. Il s'agit du modèle de la « roue de la distribution» (MCNAIR, 1958) et de la «théorie de l'accordéon » (Hollander, 1966).

Selon McNair, l'innovation dans les formats de magasins suit une trajectoire circulaire dans laquelle les «formats en place» sont concurrencés par des «formats nouveaux », moins coûteux car gérés selon une logique de rationalisation des installations, de restriction forte des assortiments et de réduction des services.

Les ressorts de la roue de la distribution peuvent ainsi être résumés de la manière suivante:

1) tous les formats de distribution nouveaux apparaissent sous une forme «bon marché». Leur succès tient essentiellement aux bas prix qu'ils pratiquent, ces bas prix étant le résultat d'une politique d'assortiments et de services réduits, visant des volumes de vente élevés ;

2) le succès de ce format initial attire des concurrents. Pour leur faire face, ce format aura tendance à mettre en œuvre des stratégies de différenciation, qui conduiront inévitablement à l'alourdissement des charges d'exploitation, et se répercuteront sur les prix;

3) cet «embourgeoisement» de la formule amorce un mouvement de rotation de la roue. La formule quitte ainsi la position «discounter» et se trouve à la merci d'un nouvel entrant proposant une formule plus «sommaire» et moins coûteuse.

Selon une perspective voisine, la «théorie de l'accordéon» (Hollander, 1966) se propose d'expliquer la succession des formats de magasin par la structure et la dynamique des assortiments. Elle met ainsi en évidence une alternance historique (d'où l'image de l'accordéon) de formats caractérisés par un assortiment large et non spécialisé, puis de formats organisés autour d'un assortiment étroit et spécialisé.

Ces théories locales de l'innovation commerciale ne s'intéressent pas directement à la question de l'emploi. Mais, tout au moins au niveau microéconomique, si l'on ne tient pas compte des phénomènes de compensation éventuels (en particulier des conséquences en termes de parts de marché des formules commerciales de type discount), on peut probablement associer des niveaux d'emplois à chaque position de la roue ou de l'accordéon. En effet, les versions discount sont associées à des niveaux d'emploi faibles, tandis que les versions embourgeoisées nécessitent un accroissement de l'emploi. De même, dans sa position «assortiment étroit et spécialisé», l'accordéon traduit des formats «destructeurs 
d'emplois », tandis que dans sa position «assortiment large non spécialisé», l'accordéon correspond à des formats «créateurs d'emplois».

\section{Les typologies de l'innovation dans les services issues d'investigations empiriques}

Les approches dites servicielles ont multiplié les investigations empiriques afin de mettre en évidence l'existence de formes particulières d'innovation dans les services. Ces investigations dénoncent unanimement une certaine myopie des conceptions technologistes, qui privilégient l'innovation technologique au détriment d'autres formes d'innovation.

Des travaux de ce type ont été réalisés pour différentes catégories de service: services de conseil, services financiers et d'assurance, services de distribution, hôtellerie-restauration, services de proximité, etc. La question de l'emploi dans sa relation à l'innovation y est rarement envisagée. Ce n'est pas surprenant, dans la mesure où les efforts de recherche sont concentrés sur la question difficile de la nature hétérogène de l'innovation, associée à la nature hétérogène du produit. En effet, les types de «produits» sont extrêmement variables d'une activité de service à l'autre. Ainsi, l'innovation de produit n'a pas le même contenu pour un service hôtelier, un service de conseil ou un service financier. La difficulté d'application des définitions traditionnelles semble augmenter avec les services dont le support est l'information, la connaissance ou l'individu.

Cependant, il est possible de faire des hypothèses sur la capacité à créer ou à détruire des emplois des différentes formes d'innovation mises en évidence dans la littérature servicielle, en prenant comme point de référence la distinction traditionnelle entre l'innovation de produit et l'innovation de process. En effet, rappelons-le, toutes choses égales par ailleurs, c'est-à-dire sans tenir compte des phénomènes de compensation positifs ou négatifs (cannibalisation des produits anciens par les innovations de produit, effet prix pour les innovations de process, etc.), on admet généralement que les innovations de produit sont favorables à l'emploi dans la mesure où elles augmentent la variété des biens et des services et où elles ouvrent de nouveaux marchés. Les innovations de process quant à elles sont destructrices d'emplois (au niveau de la firme tout au moins et à un moment donné) dans la mesure où elles visent à améliorer l'efficacité de la production de biens ou de services, donc à substituer du capital au travail. On peut tenter d'évaluer les potentiels «créateurs d'emplois» ou «destructeurs d'emplois» d'un certain nombre de formes particulières d'innovation proposées dans la littérature servicielle. Dans les analyses qui suivent, l'enjeu théorique principal n'est pas, à ce stade, il faut le noter, la question de l'impact sur l'emploi (puisque nous partons de l'hypothèse que, toutes choses égales par ailleurs, les innovations de produit et les innovations de process engendrent respectivement des effets créateurs et des effets destructeurs d'emplois), mais celle de l'identification et de la désignation du produit et du process. Les formes d'innovation proposées par la littérature servicielle contournent le plus souvent la distinction innovation de produit-innovation de process, mais on peut tenter d'apprécier lequel des effets «produit» ou «process» semble dominer dans chaque type d'innovation. Nous allons tenter de réaliser cet exercice pour trois typologies de l'innovation associées à trois secteurs de services différents.

\section{Les services intensifs en connaissances (SIC)}

Sur la base d'une investigation empirique approfondie dans de nombreuses firmes de conseil appartenant à différents secteurs, Gallous $(1994,2002)$ propose de distinguer trois types d'innovation: l'innovation ad hoc, l'innovation de «nouveau champ d'expertise », l'innovation de formalisation.

- L'innovation ad hoc est définie comme une solution permettant d'éclairer, avec un certain degré de nouveauté, le problème (juridique, organisationnel, stratégique, technique...) d'une firme sans qu'il soit nécessairement possible de la transférer (totalement) à d'autres (GALlouj, 2002). Il s'agit en quelque sorte d'une innovation incrémentale de produit dans laquelle le produit n'est pas une entité tangible, mais une solution intellectuelle à un problème d'entreprise. On peut donc faire l'hypothèse que, toutes choses égales par ailleurs, c'est ici l'effet «produit» («créateur d'emplois ») qui intervient, dans la mesure où l'innovation ad hoc (et cette aptitude à produire des solutions innovantes) est à l'origine de la conquête de nouveaux clients et du renouvellement des demandes des clients actuels. On fait ainsi l'hypothèse que le recours au conseil s'explique davantage par les niveaux d'expertise des consultants que par le coût du conseil.

- L'innovation de «nouveau champ d'expertise» décrit l'accumulation de connaissances-input relatives à des champs de connaissances émergents (Internet en est aujourd'hui un bel exemple), avec l'idée de fournir des prestations (connaissances-output) relatives à ces champs nouveaux (Gallouj, 2002). Par analogie avec les catégories de SCHUMPETER (1961), et dans la mesure où, pour les SIC, la connaissance est à la fois l'input et l'output, on peut dire que l'innovation de nouveau champ d'expertise recouvre à la fois 1) de nouveaux produits;2) de nouveaux marchés; 3) de nouvelles sources de matières premières. Ce type d'innovation, contrairement au précédent, est radical. C'est de nouveau ici l'effet «produit» («créateur d'emplois ») qui est clairement dominant. L'innovation de «nouveau champ d'expertise» peut se traduire par la création de départements nouveaux dans les firmes existantes, voire par la création d'entreprises nouvelles. 
- L'innovation de formalisation désigne un ensemble hétérogène de mécanismes qui visent à rendre le service moins flou, moins «immatériel». Ce objectif peut être atteint par deux types de mécanismes différents qui peuvent se conjuguer: 1) des mécanismes tangibles: par exemple, l'introduction de systèmes techniques dans la formule de service; 2) des mécanismes intangibles: l'introduction de méthodes, c'est-à-dire de «scripts» décrivant la distribution des rôles dans cette véritable « représentation » (théâtrale) qu'est la prestation de service; la conception et l'usage de boîtes à outils comportant des instruments analytiques structurant les raisonnements et les comportements (par exemple, les matrices du BCG); la mise en place d'une organisation qui incorpore le service immatériel.

Il serait erroné d'envisager cette forme d'innovation, par opposition aux deux formes d'innovation (de produit) précédentes, comme une innovation de processus, autrement dit, de réduire la formalisation à une activité de rationalisation. L'innovation de «formalisation» a un impact probablement indéterminé sur l'emploi ou plus exactement un impact variable, dépendant du type de formalisation réalisé. On pourrait considérer grossièrement que les mécanismes tangibles exercent plutôt un effet «process», tandis que les mécanismes intangibles exercent un effet «produit». Autrement dit, si elle consiste en l'introduction de systèmes techniques, alors la formalisation génère des économies de main-d'œuvre (effet process), mais si elle revêt des formes immatérielles (visant à rendre le produit plus visible sans effet significatif sur l'efficacité de sa réalisation), alors c'est l'effet produit («créateur d'emplois ») qui est dominant. Mais en réalité, certains mécanismes tangibles génèrent également des effets «produit», alors que l'effet «process» est également présent dans les mécanismes intangibles.

\section{Les services d'assurance et les services financiers}

Dans l'assurance et les services financiers, la typologie proposée (GADREY, GALlouJ, 1994) peut paraître a priori simple et facile à interpréter, dans la mesure où elle dissocie pour l'essentiel des innovations de produit et des innovations de process, qui sont ainsi désignées ( $c f$. tableau 3 ). Cependant, l'exercice d'interprétation est plus difficile qu'il n'y paraît. En effet, pour faciliter l'analyse, les différents types d'innovations sont présentés ici de manière séparée. En réalité, ils sont bien souvent indissociables dans leur production comme dans leurs effets. Ainsi, de nombreuses innovations de process et de produit ne sont que les deux facettes d'un même phénomène et les innovations de process et d'organisation sont souvent indissociables. Autrement dit, une même innovation (ou convention d'innovation) est le terrain d'influences contradictoires en termes de création d'emplois, dont il n'est pas aisé d'effectuer le bilan.
Les catégories A, B, C (innovations de produitsservices, innovations architecturales, innovations de modification du produit-service) sont néanmoins bien des innovations de produits d'un type certes particulier, puisqu'elles sont immatérielles (nouveaux contrats, nouveaux services). Elles constituent le cœur de l'activité d'assurance, mais échappent pour l'essentiel, par exemple, au modèle de BARrAs (1986), qui retient, comme nous l'avons déjà souligné, une définition très restrictive des «nouveaux produits». Leur effet sur l'emploi est ainsi a priori (toutes choses égales par ailleurs) de type «créateur d'emplois». En revanche, dans les catégories D1, D2, D3, D4 (innovations de process, d'organisation, de méthodes et de gestion), c'est l'effet de proccess («destructeur d'emplois») qui est mis en valeur. Néanmoins, il est clair que les effets des différentes innovations sur l'entreprise, ses métiers, ses qualifications, ses emplois, etc. ne peuvent être isolés, mais doivent être appréhendés de manière systémique.

\section{Les services de distribution}

Dans les services de distribution, DupuIs (1998) propose de distinguer les quatre formes suivantes d'innovation: 1) l'innovation de concept; 2) l'innovation de flux; 3) l'innovation organisationnelle; 4) l'innovation architecturale.

L'innovation de concept peut être définie comme la combinaison d'un format de distribution et d'un positionnement différenciateur. C'est ainsi l'effet «produit» qui y domine, et donc, au niveau microéconomique, l'effet «créateur d'emplois».

Les innovations de flux visent à réduire les coûts en optimisant différents types de flux (physiques, financiers ou d'informations). De même, les innovations organisationnelles (internes ou externes) ont pour objectifl'amélioration du fonctionnement et de la rentabilité des réseaux. Si l'on neutralise les effets de compensations éventuels, les innovations de flux et les innovations organisationnelles exercent donc essentiellement un effet «destructeur d'emplois ».

Les innovations architecturales, enfin, sont définies comme la combinaison cohérente des différents types précédents d'innovation (de concept, de flux et organisationnelle). Leur effet sur l'emploi est ainsi difficile à déterminer a priori dans la mesure où il combine et est la résultante des effets destructeurs et créateurs d'emplois.

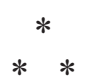

La question de l'innovation dans les services dans ses relations à l'emploi est une question fondamentale, qui paradoxalement n'occupe pas la place qui lui revient dans la littérature économique. Dans cet article, nous avons, à titre essentiellement programmatique, envisagé cette question, en tentant de l'ar- 
Tableau 3

Les principales formes d'innovation dans l'assurance

\begin{tabular}{|c|c|c|c|}
\hline $\begin{array}{l}\text { Types } \\
\text { d'innovation }\end{array}$ & \multicolumn{2}{|c|}{ Sous catégories } & Définition \\
\hline \multirow{4}{*}{$\begin{array}{l}\text { A : } \\
\text { Innovations } \\
\text { de « produits- } \\
\text { services » }\end{array}$} & \multicolumn{2}{|c|}{ A1 : Innovations de produits-services } & Service, concept, contrat, nouveau \\
\hline & \multirow{3}{*}{$\begin{array}{l}\text { A2 : Innovations } \\
\text { de produits- } \\
\text { services sur } \\
\text { mesure }\end{array}$} & $\begin{array}{l}\text { 1) Innovations sur mesure } \\
\text { d'adaptation }\end{array}$ & Adaptation à un client d'un contrat standard \\
\hline & & $\begin{array}{l}\text { 2) Innovations «sur mesure } \\
\text { total» }\end{array}$ & $\begin{array}{l}\text { Conception d'un contrat réellement spécifique pour un client } \\
\text { donné }\end{array}$ \\
\hline & & $\begin{array}{l}\text { 3) Couverture de risques } \\
\text { spéciaux }\end{array}$ & $\begin{array}{l}\text { Couverture d'un risque nouveau concernant des populations } \\
\text { statistiques réduites }\end{array}$ \\
\hline \multirow{2}{*}{$\begin{array}{l}B \text { : Innovations } \\
\text { architecturales }\end{array}$} & \multicolumn{2}{|c|}{$\begin{array}{l}\text { B1 : Innovation d'association de « produit- } \\
\text { services » }\end{array}$} & Combinaison de produits-services existants \\
\hline & \multicolumn{2}{|c|}{$\begin{array}{l}\text { B2 : Innovation de dissociation de « produit- } \\
\text { services » }\end{array}$} & $\begin{array}{l}\text { Isolation d'un fragment de produit-service pour la vente } \\
\text { séparée }\end{array}$ \\
\hline \multicolumn{3}{|c|}{$\mathrm{C}$ : Innovations de modification du produit-service } & $\begin{array}{l}\text { La formule de base est inchangée, mais certaines spécifica- } \\
\text { tions ou options changent }\end{array}$ \\
\hline \multirow{4}{*}{$\begin{array}{l}\text { D: } \\
\text { Innovations } \\
\text { de process, } \\
\text { d'organisation, } \\
\text { de méthodes et } \\
\text { de gestion }\end{array}$} & \multicolumn{2}{|c|}{$\begin{array}{l}\text { D1 : Innovations accompagnant les innovations } \\
\text { de produits-services }\end{array}$} & $\begin{array}{l}\text { Innovation de process et d'organisation consécutive à une } \\
\text { innovation de produits-services des types A, B ou C et indis- } \\
\text { sociable d'elles }\end{array}$ \\
\hline & \multicolumn{2}{|c|}{$\begin{array}{l}\text { D2 : Innovation pour un produit-service identique } \\
\text { dans ses spécifications formelles et dans son } \\
\text { mode de prestation }\end{array}$} & $\begin{array}{l}\text { Modification importante du processus (technologie, organi- } \\
\text { sation du travail) pour un service final inchangé }\end{array}$ \\
\hline & \multicolumn{2}{|c|}{$\begin{array}{l}\text { D3 : Innovations pour un produit-service iden- } \\
\text { tique dans les spécifications formelles mais } \\
\text { amélioré dans la réalisation concrète, la qualité } \\
\text { perçue, le mode de commercialisation }\end{array}$} & $\begin{array}{l}\text { Modification importante du processus (technologie, organi- } \\
\text { sation du travail) pour un produit « formellement » identique } \\
\text { mais de meilleure qualité }\end{array}$ \\
\hline & \multicolumn{2}{|c|}{ D4 : Innovations de gestion } & $\begin{array}{l}\text { Innovations portant sur la gestion financière, actuarielle, } \\
\text { juridique, des ressources humaines, etc. }\end{array}$ \\
\hline
\end{tabular}

ticuler aux principaux travaux empiriques ou théoriques consacrés à l'innovation dans les services. $\mathrm{Si}$, bien qu'elle soit unanimement considérée comme fondamentale, cette question est relativement peu exploitée, c'est que l'une des variables de la relation (à savoir la question de l'innovation dans les services) n'est pas encore clairement élucidée sur le plan théorique. En effet, le caractère encore flou de la nature et du statut de l'innovation dans les services conduit à considérer comme hasardeuse toute tentative d'analyse des conséquences de cette innovation sur l'emploi.

Il s'agit-là pourtant d'un champ de recherche prometteur, important sur le plan théorique et des politiques publiques qu'il faudrait explorer davantage, à la fois sur le plan micro, méso et macroéconomique, que ce soit par le biais de la construction théorique déductive ou de l'analyse inductive qualitative ou statistique. Les deux axes généraux de recherche que nous avons explorés dans cet article peuvent fournir un premier cadrage pour une telle réflexion.

Plusieurs autres champs intéressants, que nous n'avons pas évoqués ici, mériteraient une analyse spécifique. Il s'agit, tout d'abord, de la question de l'innovation non plus dans les services, mais par les services, c'est-à-dire du rôle que peuvent jouer certaines activités de services (en particulier les
SIC) dans l'innovation et l'emploi de leurs clients. Il n'existe pas, à notre connaissance, de travaux statistiques consacrés au lien entre l'innovation induite par les SIC et l'emploi chez leurs clients. C'est une piste de recherche intéressante à explorer, qu'il faudrait envisager en construisant des questionnaires spécifiques.

Il s'agit ensuite de la question de l'entrepreneuriat dans les services dans ses relations à l'emploi. En effet, si la question de l'entrepreneuriat dans ses relations aux services n'est pas nouvelle, dans la mesure où l'essentiel des créations d'entreprises s'opère dans le secteur tertiaire, en revanche, celle de l'entrepreneuriat dans sa relation à l'innovation dans les services est rarement envisagée.

On peut également aborder la question de la relation innovation emploi en inversant les termes de la causalité, c'est-à-dire en se consacrant à l'analyse des conséquences de la nature du marché du travail sur l'innovation et la productivité. Ainsi, sans s'intéresser, il est vrai, aux services exclusivement, KLEINKNECHT et alii (2006) montrent que la flexibilisation (externe) des marchés du travail a des effets négatifs importants sur les activités d'innovation au sein des firmes, dans la mesure où elle dégrade la confiance et la loyauté nécessaires au développement de l'innovation et à l'amélioration de la productivité. 


\section{Bibliographie}

Abernathy W., Utterback J. (1978), "Patterns of industrial innovation", Technology Review, 80, p. 41-47.

Amable B., Barré R., Boyer R. (1997), Les systèmes d'innovation à l'ère de la globalisation, Economica.

Appelbaum E. (1987), “Technology and the redesign of work in the insurance industry", in Drygulski White B. (ed) Women, work and technology. Transformations, University of Michigan Press.

BARRAS R. (1986), "Towards a theory of innovation in services", Research Policy, 15, p. 161-173.

BAumOL W. (1967), "Macroeconomics of unbalanced growth", Amercian economic review, 2, 415-426.

Bell D. (1976), Vers la société post-industrielle, Laffont.

Bertrand O. (1988), «Qualité et hétérogénéité des emplois de services », Formation et Emploi, n 23, p. 1929.

Bertrand O. Noyelle T. (1987), «L'emploi dans les banques et assurances: comparaisons internationales et perspectives d'évolution», Économie et Humanisme, $\mathrm{n}^{\circ} 295$.

Bluestone B., Harrison B. (1986), The Great American Job Machine, Report for the Joint Economic Committee, December.

Boyer R. (2002), La croissance, début de siècle: de l'octet au gène, Albin Michel.

Cossalter C., Hezard L. (1983), Nouvelles perspectives de l'informatisation dans les banques et les assurances, CEREQ.

Devetter F.-X. (2001), L'économie de la disponibilité temporelle au travail, thèse de doctorat, université de Lille 1.

Djellal F. (2002), "Innovation trajectories in the cleaning industry", New Technology and Employment, vol. 17, $\mathrm{n}^{\circ} 2$, p. 119-131.

Duellal F., Francoz D., Gallouj C., Gallouj F., Jacquin Y. (2003), "Revising the definition of research and development in the light of the specificities of services", Science and Public Policy, 30/6, p. 415-430.

Djellal F., Gallouj F. (2000), «Le casse-tête» de la mesure de l'innovation dans les services: enquête sur les enquêtes ", Revue d'économie industrielle, $\mathrm{n}^{\circ} 93,4 \mathrm{e}$ trimestre, p. 7-28.

Dupuis M. (1998), «L'innovation dans la distribution: ses implications dans les rapports industrie-commerce», Décision Marketing, n 15(3), p. 29-41.

Evangelista R. (2000a), Innovation and Employment in Services: Results from the Italian Innovation Survey, in
Vivarelli M., Pianta M. (eds) The Employment Impact of Innovation: Evidence and Policy, Routledge.

EVANGELISTA R. (2000b), "Sectoral patterns of technological change in services", Economics of innovation and new technology, 9, p. 183-221.

Evangelista R., Savona M. (2003), "Innovation, employment and skills in services: firm and sectoral evidence", Structural Change and Economic Dynamics, 14, p. 449474.

Kleinknecht A., Oostendorp R.M., Pradhan M.P., NAAstepad C.W.M. (2006), "Flexible Labour, Firm Performance and the Dutch Job Creation Miracle", International Review of Applied Economics, vol. 20, $\mathrm{n}^{\circ} 2$, p. 171-187.

Fourastié J. (1949), Le grand espoir du XXe siècle, PUF.

Freeman C., Soete L. (1994), Work for all or mass unemployment? computerised technical change into the twenty-first century, Pinter.

Freeman C., Soete L. (eds) (1987), Technical change and full employment, Basil Blackwell.

Freeman C., Clark J., Soete L. (1982), Unemployment and technical innovation, Pinter.

GADREY J. (2005), Les quatre «mondes» des économies de services développées, Économies et Sociétés, $\mathrm{n}^{\circ} 11-$ 12/2005, p. 1925-1970.

Gadrey J., Gallouj F. (1994), L'innovation dans l'assurance: le cas de l'UAP, UAP et ministère de l'Enseignement supérieur, de la Recherche et de la Technologie.

Gallouj F. (1994), Économie de l'innovation dans les services, L'Harmattan.

Gallouj F. (2002), Innovation in the Service Economy: the New Wealth of Nations, Edward Elgar Publishers.

Hollander S.C. (1966), Notes on the retail accordion, Journal of Retailing, 42(2), p. 24-34.

JANY-CATRICE F. (2004), «Une analyse socio-économique de l'emploi dans l'hôtellerie-restauration en France et aux États-Unis», Économies et sociétés, série Socioéconomie du travail, $\mathrm{n}^{\circ} 23$ (1).

Katsoulacos Y. (1984), "Product innovation and employment”, European Economic Review, 26, p. 83-108.

MAHAR, M. (1992), "Blue collar, white collar: good jobs are vanishing throughout the economy", Barron's, May, 11, 8-24.

McNaIR, M.P. (1958), "Significant trends and developments in the post war period", in A.B. Smith (ed.) Competitive Distribution in a Free High Level Economy 
and its implication for the University, University of Pittsburgh Press, p. 1-25.

NäHlinder J., Hommen L. (2002), Employment and Innovation in Services: Knowledge Intensive Business Services in Sweden, Report for the final meeting and conference of AITEG, Birkbeck College, Londres, 18 au 18 avril.

OCDE (1995) Manuel sur la mesure des ressources humaines consacrées à la science et à la technologie, Manuel de Canberra, Paris.

OCDE (1997), Principes directeurs proposés pour le recueil et l'interprétation des données sur l'innovation technologique, Manuel d'Oslo, Paris.

PavitT K. (1984), Sectoral "Patterns of Technical Change: Towards a Taxonomy and a Theory", Research Policy, $\mathrm{n}^{\circ} 13$, p. 343-373.

Peters B. (2004), Employment effects of different innovation activities : microeconometric evidence, $\mathrm{ZEW}$, dicussion paper $\mathrm{n}^{\circ}$ 04-73.

Petit P. (1995), Employment and Technological Change, in Stoneman P. (ed) Handbook of the economics of innovation and technological change, p. 366-408.
Petit P. (2002), Growth and Productivity in a knowlege based service economy, in Gadrey J., Gallouj F. (eds) Productivity, Innovation and knowledge in services, Edward Elgar.

Petit P., Soete L. (eds) (2001), Technology and the future of european employment, Edward Elgar.

SChumpeter J. (1961), Théorie de l'évolution économique, Librairie Dalloz, Paris.

Soete L., Miozzo M. (1990), Trade and Development in Services: a Technological Perspective, mimeo, MERIT.

Thurow L. (1989), Towards a high-wage, high productivity service sector, Economic Policy Institute, Washington DC.

Vivarelli, Pianta (eds), (2000), The employment impact of innovation: evidence and policy, Routledge.

Vivarelli M. (1995), The economics of technology and employment: Theory and Empirical Evidence, Edward Elgar.

WeBster J. (1996), Shaping women's work. Gender employment and information technology, Longman. 